\title{
Herpes simplex virus 2 causes apoptotic infection in monocytoid cells
}

\author{
Antonio Mastino ${ }^{1,4}$, Maria Teresa Sciortino', \\ Maria Antonietta Medici ${ }^{1}$, Donata Perri ${ }^{1}$, \\ Maria Grazia Ammendolia ${ }^{1}$, Sandro Grelli ${ }^{2}$, Carla Amici ${ }^{3}$, \\ Antonio Pernice ${ }^{1}$ and Salvatore Guglielmino ${ }^{1}$ \\ 1 Institute of Microbiology, Faculty of Sciences, University of Messina, Messina, \\ Italy \\ 2 Institute of Experimental Medicine, C.N.R., Rome, Italy \\ ${ }^{3}$ Department of Experimental Medicine and Biochemical Sciences, University of \\ Rome 'Tor Vergata', Rome, Italy \\ ${ }^{4}$ corresponding author: Prof. Antonio Mastino, Department of Experimental \\ Medicine and Biochemical Sciences, University of Rome 'Tor Vergata', via di \\ Tor Vergata 135, 00133 Rome, Italy; tel: +39 6 72596592; fax: +39 620427282
}

Received 10.2.97; revised 23.5.97; accepted 12.6.97

Edited by L. Fesus

\begin{abstract}
Increasing evidence indicates that apoptosis can be associated with several viral infections. Here we demonstrate, that infection of monocytoid cells by Herpes simplex virus 2 (HSV-2) resulted, in time- and dose-dependent induction of apoptosis as an exclusive cytopathic effect. The phenomenon was confirmed using four different techniques. Conversely, apoptosis was not observed in the Vero cell line. Virus yield in monocytoid cells was delayed and reduced, although well detectable, in comparison with that observed in Vero cells. Nevertheless, released virions exhibited full infecting capability. Apoptosis induced by HSV-2 was not inhibited by cycloheximide and only partially by an UV-treatment which completely abrogated infectivity. Virus-induced apoptosis was partly inhibited by indomethacin and was associated with a down-regulation of Bcl-2. A similar, but less pronounced, apoptosis-inducing effect in monocytoid cells was also observed with HSV-1 infection. Depending on the target cells, therefore, HSV could complete a cycle of infection which is characterized by apoptosis of infected cells.
\end{abstract}

Keywords: apoptosis; Herpes simplex virus; monocytoid cells; viral infection

Abbreviations: HSV-2, herpes simplex type 2; HSV-1, herpes simplex type 1; MOI, multiplicity of infection; PFU, plaque forming unit; FCS, fetal calf serum; CM, complete medium; $\mathrm{CHX}$, cycloheximide

\section{Introduction}

Mechanisms involved in the cytopathic effect of viruses during infection are largely unknown. Viruses were considered to cause cell death by destroying cell membrane, by cell fusion, or by lethal damage of host cell transcriptional or translational machinery. As a consequence, cell necrosis, which can result from these events during productive replication, was supposed, in the past, to be the exclusive form of cell death associated with viral infection. However, increasing evidence suggets that apoptosis, the cellular mechanism of selfdestruction triggered by either physiological or pathological stimuli (Kerr et al, 1972; Arends and Wyllie, 1991), may occur during several viral infections, including those caused by members of the Herpesviridae family (reviewed in McCabe and Orrenius, 1992; Razvi and Welsh, 1995; Shen and Shenk, 1995). In particular, herpes simplex virus 1 (HSV-1), a member of Alphaherpesvirinae subfamily, has been associated, similarly to members of other virus families, with both apoptosis-inducing and apoptosis-preventing actions (Chou and Roizman, 1992; Tropea et al, 1995). The different fate of target cells has recently been attributed to whether or not the virus is able to express genes codifying for proteins exerting anti-apoptotic actions (Leopardi and Roizman, 1996). Herpes simplex virus 2 (HSV-2), is another member of the Alphaherpesvirinae subfamily of herpesviruses, characterized by a variable host range, a short replicative cycle and rapid cell to cell spread in culture. Moreover, infection by HSV2 is characterized, similarly to that caused by HSV-1, by the ability of the virus to establish a wide spectrum of relationships with infected tissues, ranging from a latent form of silent infection to rapid induction of death in infected cells (Roizman and Sears, 1996).

In the present study we have investigated the role of the cellular target in the processes which may or may not lead to cell death by apoptosis in infections caused by herpes simplex viruses. To this purpose we have principally used the in vitro infection of human monocytoid cells with HSV-2, compared to that of fully permissive Vero cells, as an experimental model.

\section{Results}

Herpes simplex virus type 2 induces apoptosis of monocytoid cells

Results showed a marked increase in the percentage of apoptotic cells following HSV-2 infection, when compared with control uninfected or mock-infected cells, using morphological analysis by light microscopy and acridine orange or Hoechst staining. In the experiment shown in Figure 1, the level of apoptosis reached about $33 \%, 48 \mathrm{~h}$ post infection (p.i.) (Figure $1 \mathrm{~b}$ and d). Uninfected controls showed a low level of spontaneous apoptosis ( $<2 \%$ ) (Figure 1a and $c$ ), as did mock-infected cells (data not shown). In order to confirm the above-reported results, we utilized flow cytometry analysis of isolated nuclei, a highly reproducible and 

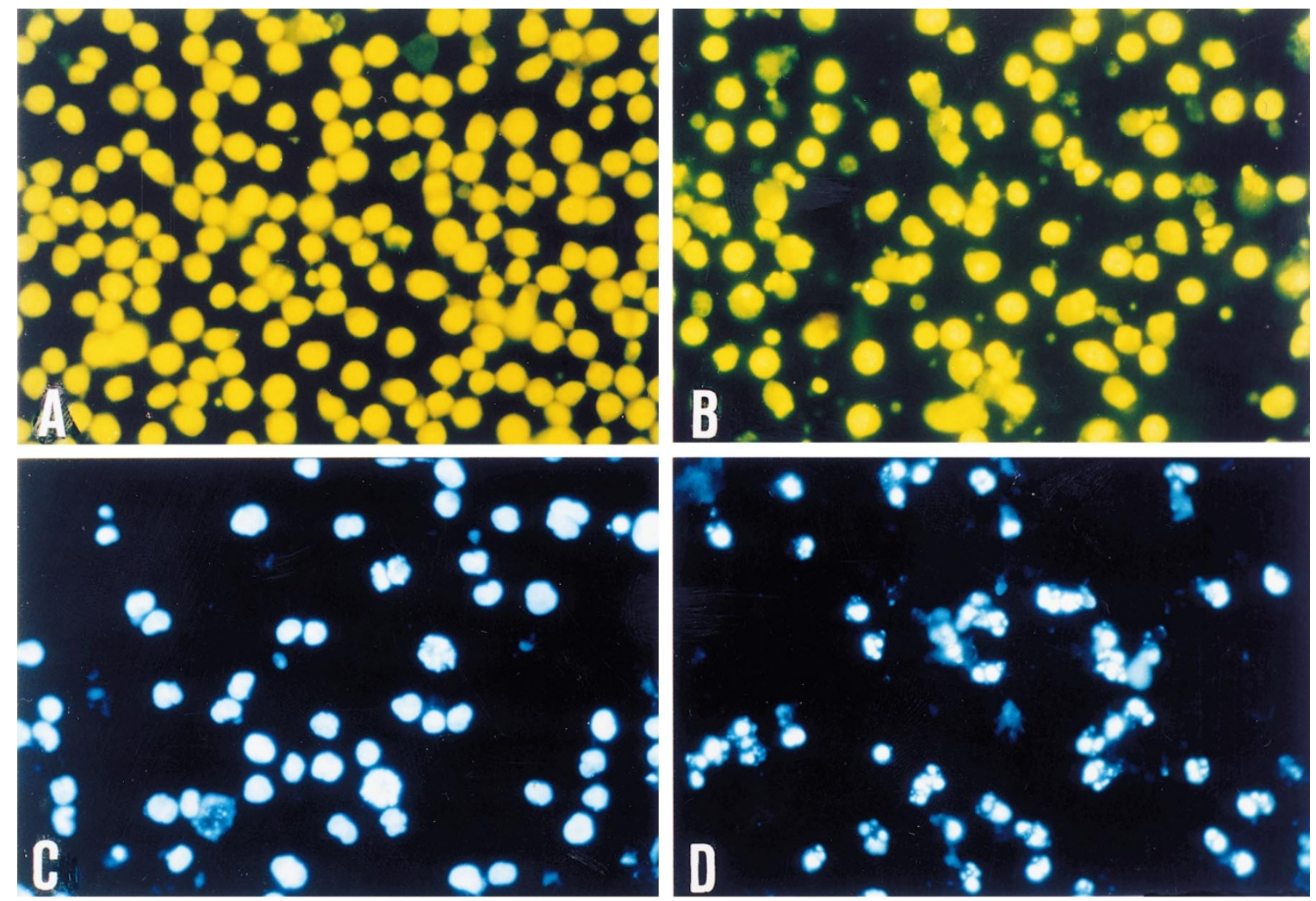

Figure 1 Fluorescence microscopy analysis of HSV-2-infected U937 cells. Control cells (A, C) and cells infected with HSV-2 at a MOI of 10 PFU/cell (B, D) were analyzed $48 \mathrm{~h} \mathrm{p.i.} \mathrm{after} \mathrm{staining} \mathrm{with} \mathrm{the} \mathrm{fluorescent} \mathrm{DNA-binding} \mathrm{dyes} \mathrm{acridine} \mathrm{orange} \mathrm{(A,} \mathrm{B)} \mathrm{or} \mathrm{Hoechst} \mathrm{(C,} \mathrm{D).} \mathrm{In} \mathrm{infected} \mathrm{samples} \mathrm{(B,} \mathrm{D),} \mathrm{many} \mathrm{cells} \mathrm{show} \mathrm{typical}$ characteristics of advanced apoptosis with nuclei present as one or more groups of featureless, bright, spherical beads. Magnification $(\times 400)$

quantitative technique to detect apoptosis. Results unconfutably confirmed morphological analysis. In the experiment shown in Figure 2, the percentage of hypodiploid nuclei obtained from control cells, after $48 \mathrm{~h}$ of culture, was still rather low (4\%, Figure $2 \mathrm{a})$, while hypodiploid nuclei obtained from cells infected with HSV-2 with a multiplicity of infection (MOI) of 10 plaque forming units (PFU)/cell rose to $71 \%$ (Figure 2b). Apoptosis in U937 cells infected with HSV-2 was also evaluated by using the TUNEL technique, an assay able to directly detect DNA fragmentation at single cell level. In the experiment illustrated in Figure 2 (from $\mathrm{c}$ to $\mathrm{f}$ ), $48 \mathrm{~h}$ after infection we obtained $26 \%$ positive apoptotic cells from the culture infected at a $\mathrm{MOI}$ of $1 \mathrm{PFU} /$ cell (Figure $2 \mathrm{e}$ ) and $61 \%$ positive apoptotic cells from the culture infected at a MOI of 10 PFU/cell (Figure 2f), in comparison with $14 \%$ positive cells from the mock-infected culture (Figure 2c) and $96 \%$ in the positive control consisting of DNase I-treated cells (Figure 2d).

\section{Kinetic, dose-dependency of herpes simplex type 2-induced apoptosis and comparison with herpes simplex type 1}

The induction of apoptosis in U937 by HSV-2 was dependent on the dose of virus inoculum and on the length of time of culture following infection. Table 1 summarizes the kinetic, dose-dependency and variability of the phenomenon of HSV- 2-induced apoptosis in U937 monocytoid cells, resulting from mean values of \% apoptosis plus SD obtained from three independent experiments. Apoptosis induced by HSV-2 at various PFU/cell ratios was evaluated by fluorescence microscopy analysis of cells after staining with acridine orange, as described below. At a high $\mathrm{MOI}$ we observed, within $72 \mathrm{~h}$ p.i., a level of apoptosis, comprising practically all the cells in culture. Again in Table 1, we can compare the results obtained when the ' $F$ ' strain of HSV-1 was utilized in place of HSV-2, showing a similar but less pronounced apoptosis-inducing effect.

\section{Herpes simplex type 2 does not induce apoptosis in Vero cells}

Moreover, in order to verify whether HSV-2 infection of U937 could result in complete cleavage of nuclear DNA into oligonucleosome-sized fragments, considered as the ultimate event of apoptosis, we utilized the DNA-electrophoresis-on-agarose-gel technique. Results reported in Figure 3 show the presence of the typical apoptotic 'DNA ladder' in U937 cells infected with HSV-2 at a MOI of 10 $\mathrm{PFU} /$ cell $48 \mathrm{~h}$ p.i. The same experiment showed no evidence of apoptosis in the fully permissive Vero cell line tested at different times following infection with HSV-2, extending to HSV-2 what has been recently demonstrated for HSV-1 in the same cell line (Leopardi and Roizman, 
1996). The resistance of Vero cells to HSV-2-induced apoptosis was confirmed by repeated failure to detect any evidence of apoptosis, using all the above reported techniques (data not shown). Unexpectedly, in some preliminary experiments using a U937 cell line of different origin in respect to that utilized in all the experiments shown in this report, while all other assays showed the presence of apoptotic cells in cultures infected with HSV-2, only the gel electrophoresis technique did not give the same result (data not shown). We were unable to understand the exact reasons for this discrepancy, but they are probably related to a characteristic peculiar to the cell line utilized, as previously described for U937 following a different inducing stimulus (Ghibelli et al, 1995). However, no evidence of cell death other than optosis was observed in cultures of U937 cells infected with herpes simplex viruses and analyzed in the above-reported or in any other experiment. Moreover, time- and dosedependent induction of apoptosis by HSV-2 was also observed in HL-60 monocytoid cells (data not shown).
A

64

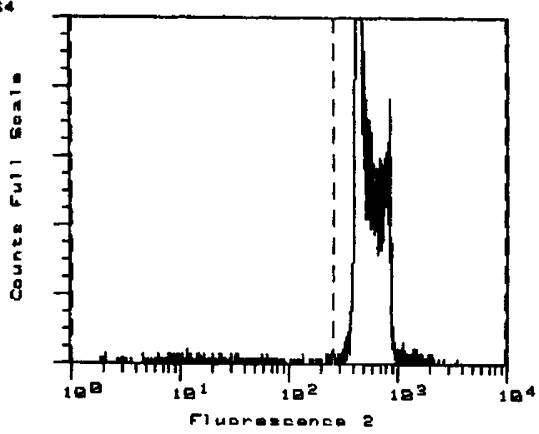

C

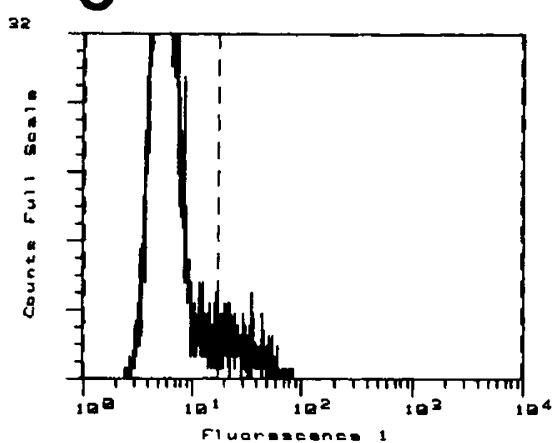

E

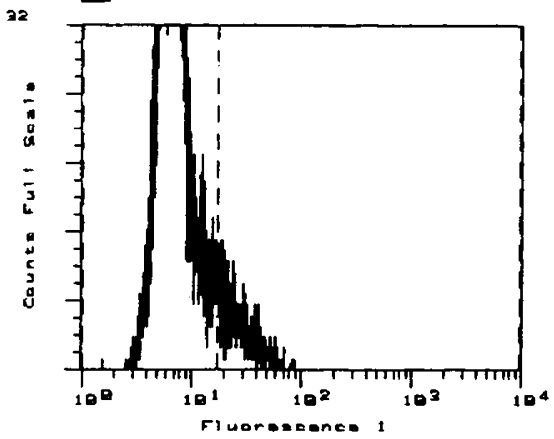

B

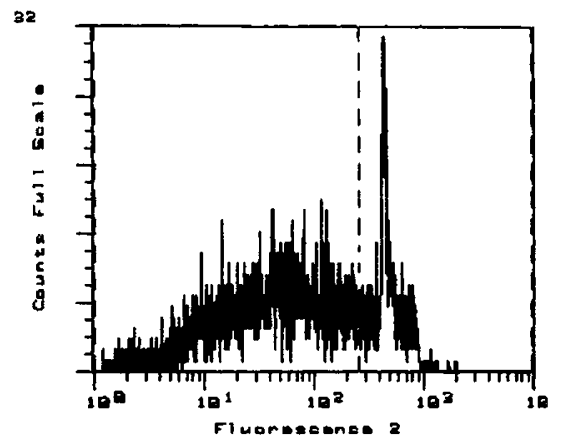

D

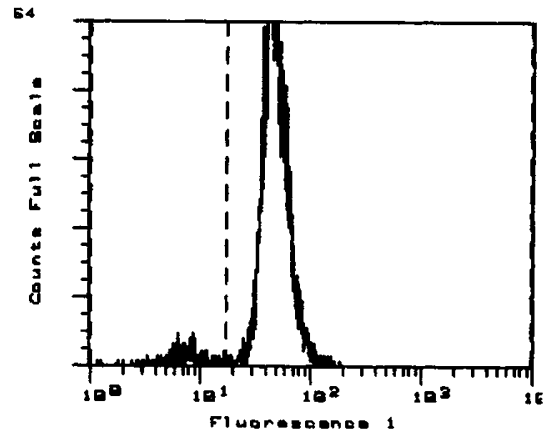

$\mathbf{F}$

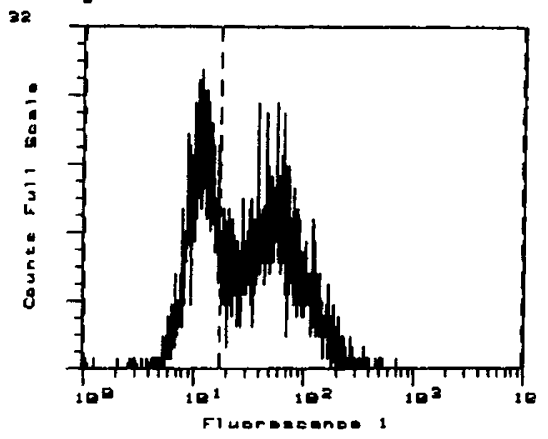

Figure 2 Induction of apoptosis by HSV-2 infection in the U937 cell line detected by flow cytometry. After 48 h of culture, nuclei from mock-infected U937 cells (A) or from U937 cells infected with HSV-2 at a MOI of $10 \mathrm{PFU} / \mathrm{cell}(\mathrm{B})$ were isolated and stained by treatment with a solution consisting of propidium iodide (50 $\mu \mathrm{g} / \mathrm{ml}$ ), sodium citrate $(0.1 \%)$ and Triton X-100 (1\%) in distilled water and analyzed by flow cytometry. The dashed lines indicate the boundaries between the peaks of hypodiploid and diploid nuclei which were arbitrarily set on the control sample and maintained in the corresponding infected sample. In another experiment DNA fragmentation was analyzed at single cel level by flow cytometry using the TUNEL technique, after $48 \mathrm{~h}$ of culture in control U937 cells (C) or in U937 cells infected with HSV-2 at a MOI of 1 PFU/cell (E) or at a MOI of 10 PFU/cell (F). Results were compared with a positive control consisting of DNase I (1 mg/ml) treated U937 cells (D). The dashed lines indicate the boundaries between the peaks of negative and positive cells which were arbitrarily set in control samples incubated with FITC-dUTP solution alone, without TdT, and maintained in all the corresponding fluorescein-dUTP/Tdt-treated samples. Detection of apoptosis using propidium iodide staining and the TUNEL technique were repeated in four and two independent experiments, respectively, with similar results 
Table 1 Induction of apoptosis in U937 cells by herpes virus infection

$\%$ apoptosis (mean $\pm S D)^{1}$

Incubation period

\begin{tabular}{lrrrr} 
Virus & PFU/cell & \multicolumn{1}{c}{$\mathbf{2 4} \mathbf{h}^{\mathbf{2}}$} & \multicolumn{4}{c}{$\mathbf{4 8} \mathbf{h}^{\mathbf{3}}$} & \multicolumn{1}{c}{$\mathbf{7 2} \mathbf{h}^{\mathbf{4}}$} \\
\hline Mock-infection & - & $1.17 \pm 0.80$ & $1.77 \pm 0.93$ & $1.53 \pm 0.47$ \\
HSV-2 & 0.1 & $2.73 \pm 0.61$ & $4.60 \pm 0.75$ & $10.70 \pm 2.29$ \\
HSV-2 & 1 & $5.30 \pm 1.75$ & $10.03 \pm 1.68$ & $31.27 \pm 4.36$ \\
HSV-2 & 5 & $9.67 \pm 3.45$ & $24.20 \pm 7.79$ & $66.20 \pm 9.90$ \\
HSV-2 & 10 & $13.10 \pm 3.73$ & $38.33 \pm 8.28$ & $87.33 \pm 3.20$ \\
HSV-2 & 20 & $21.10 \pm 1.30$ & $39.07 \pm 9.62$ & $91.67 \pm 6.66$ \\
HSV-1 & 1 & $6.79 \pm 1.70$ & $8.20 \pm 3.65$ & $11.00 \pm 5.20$ \\
HSV-1 & 10 & $14.47 \pm 4.64$ & $18.13 \pm 4.45$ & $25.33 \pm 6.12$ \\
\hline
\end{tabular}

${ }^{1}$ Cumulative data from three independent experiments. ${ }^{2} P<0.05$ by Duncan multiple range test for all comparisons vs. mock-infection except HSV-2/MOI 0.1 and HSV-2/MOI 1. ${ }^{3} P<0.05$ by Duncan multiple range test for all comparisons vs. mock-infection except HSV-2/MOI 0.1 , HSV-2/MOI 1 and HSV-1/MOI 1. ${ }^{4} P<0.05$ by Duncan multiple range test for all comparisons vs. mock-infection except HSV-2/MOI 0.1 and HSV-1/MOI 1.

\section{HSV-2-induced apoptosis in monocytoid cells is associated with productive infection and inhibition of protein synthesis, at lower levels than those observed in Vero cells}

Considering the contrast between results obtained for monocytoid cells and those obtained for the fully permissive Vero cell line, we were then interested in finding out whether cell death by apoptosis could affect viral production. In fact a previous study reported no evidence of active replication of HSV-1 in U937 within 24 h p.i. (Feng et al, 1993). Figure 4a shows a time-course for total (extracellular plus cellassociated) virus yield following exposure to HSV-2 for $1 \mathrm{~h}$ at different PFU/cell ratios, starting $24 \mathrm{~h}$ p.i.. Well-detectable levels of infectivity were observed at all PFU/cell ratios of inocula utilized. However at the higher dose of virus inoculum, i.e. at a dose able to induce a well-detectable level of apoptosis within $24 \mathrm{~h}$ p.i., virus yield did not increase with the ongoing of the culture. On the contrary, at lower doses of virus inoculum, virus yield grew exponentially with the incubation time. In addition, in the range of experimental conditions tested, independently of the MOI utilized and of the time required to obtain maximal production, virus yield reached an insuperable plateau. Figure $4 \mathrm{~b}$ shows that HSV-2 not only replicated in $U 937$ cells, but was also released in the supernatant as infectious virions. The course of the curves was very similar to that observed for total virus yield, but the absolute values of infectious particles released were at least ten times lower in comparison with total virus yield, at each point tested. Moreover, both total virus and extracellular virus yields resulted markedly lower compared to those obtained in the same experiment in Vero cells, as reported in Figure $4 a$ and $b$. In repeated experiments maximal virus titers obtained in HSV-2 infected U937, using the same or different experimental conditions such as a lower MOI, did not significantly differ from those reported in the experiment shown in Figure 4, and at least a hundred times lower in comparison with those usually obtained in Vero cells. Figure 4c shows that HSV-2 infection was associated with a clear inhibition of protein synthesis in U937 cells. However, the level of inhibition was lower in comparison with that obtained

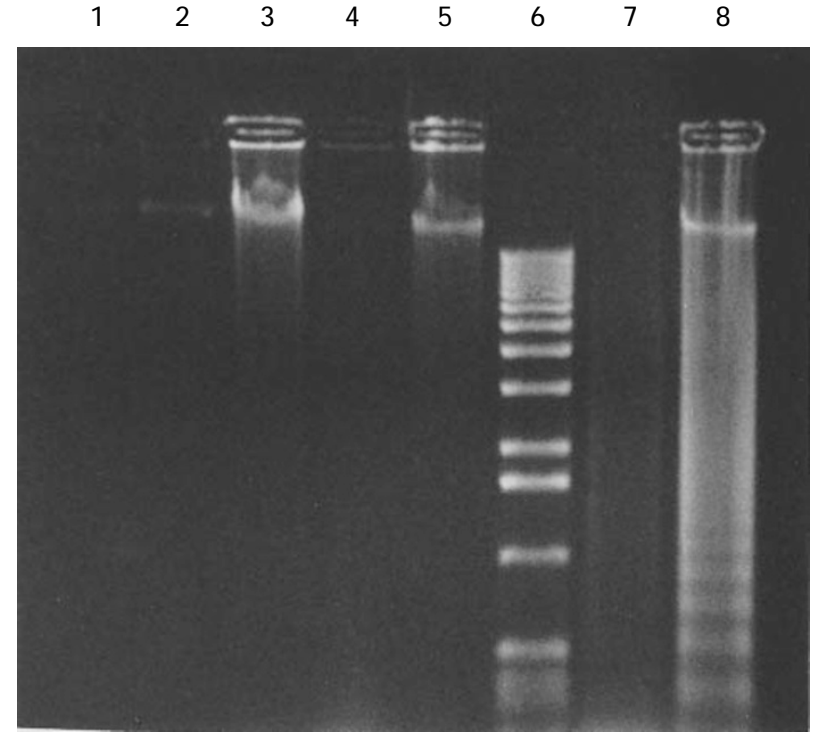

Figure 3 HSV-2 infection induces the nucleosomal ladder pattern characteristic of apoptosis, in U937 cells but not in Vero cells. U937 cells were mock-infected (lane 7) or infected with HSV-2 at a MOI of $10 \mathrm{PFU} / \mathrm{cell}$ (lane 8). After $48 \mathrm{~h}$ at $37^{\circ} \mathrm{C}$, cells were collected, DNA extracted, electrophoresed on $0.8 \%$ agarose gel and visualized by staining with ethidium bromide and by UV light transillumination. Similarly, Vero cells were mock-infected (lanes 1 and 3) or infected with HSV-2 at a MOI of 10 PFU/ cell. After $6 \mathrm{~h}$ (lane 2), $24 \mathrm{~h}$ (lane 4 ) and $48 \mathrm{~h}$ (lane 5 ) respectively at $37^{\circ} \mathrm{C}$, adherent and detached cells were collected and DNA extracted and electrophoresed as for U937 cells. Lane 6: m.w. marker of a 1-Kb ladder DNA. Vero cells did not exhibit the nucleosomal ladder pattern at any incubation time tested. Results are representative of three independent experiments

in Vero cells. The inhibition was prompt and halted about $24 \mathrm{~h}$ p.i., leveling off at approximately $50 \%$ of the control, as shown in Figure 4d. Our results do not allow us to distinguish whether this effect was due to reduced protein synthesis inhibition at single cell level or to failure to cause protein synthesis shut-off in part of the cell population. In addition, SDS-PAGE analysis did not show any appreciable differences in the pattern of viral protein synthesis, detected by comparison with uninfected counterparts, between HSV2-infected U937 and HSV-2-infected Vero cells (data not shown). Detection of large amounts of viral proteins in HSV2-infected U937 cells was obtained using HSV-2-specific monoclonal antibodies and fluorescence microscopy. However, also using this technique to detect virus replication, the percentage of specific HSV-2-protein positive cells was lower in U937 cells with respect to Vero cells, at all time p.i. tested (data not shown). Nevertheless, first appearance of positivity for HSV-2-proteins, i.e. 4-5 h p.i., was not delayed in U937 cells in comparison with that observed in Vero cells, indicating that the virus did not replicate in Vero cells faster than in U937 cells. Moreover, double fluorescence staining with anti-glycoprotein D monoclonal antibody and Hoechst, indicated that virus-specific proteins were detectable in apparently intact cells as well as in cells showing characteristic apoptotic morphology. Similarly, apoptosis was observed both in virus-protein positive and in virusprotein-negative cells (Figure 5). 
A

TOTAL VIRUS

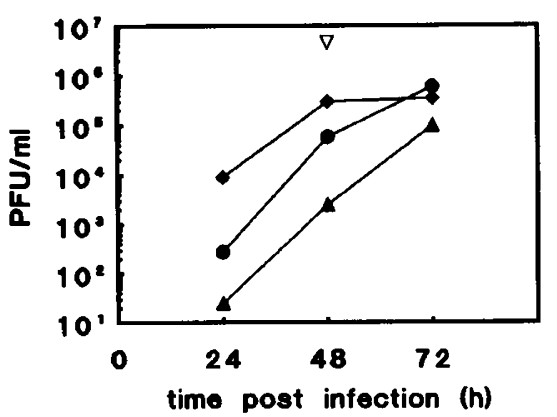

C

PROTEIN SYNTHESIS

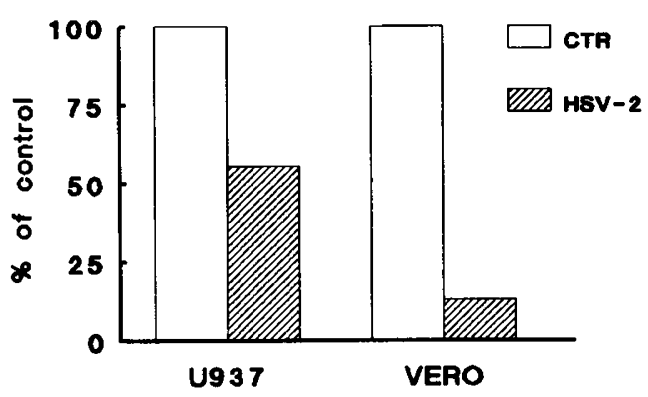

B

eXtracelluLAR VIRUS

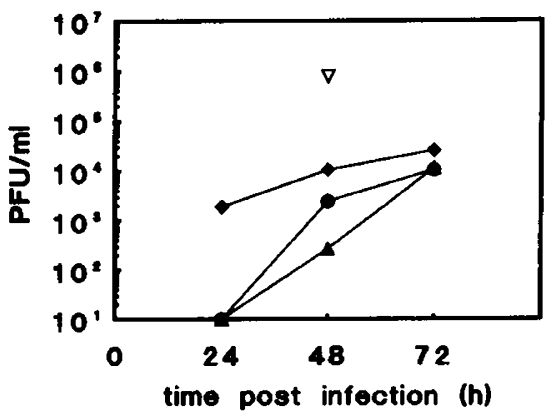

D

PROTEIN SYNTHESIS

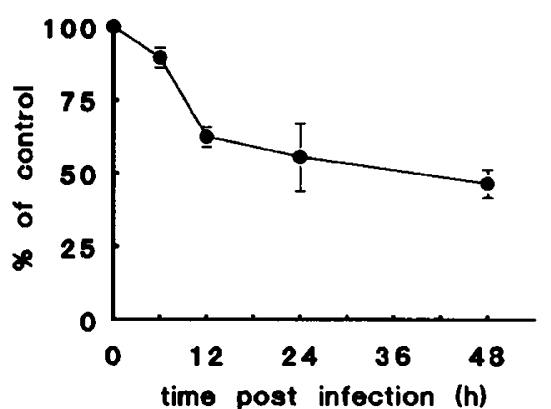

Figure 4 Effect of HSV-2 infection on virus production and on protein synthesis in U937 and in Vero cells. Virus yield was titrated by plaque assay using Vero cells, at $24 \mathrm{~h}, 48 \mathrm{~h}$ and $72 \mathrm{~h} \mathrm{p.i.,} \mathrm{from} \mathrm{U937} \mathrm{cells} \mathrm{exposed} \mathrm{to} \mathrm{HSV-2} \mathrm{at} \mathrm{a} \mathrm{MOI} \mathrm{of} 0.1 \mathrm{PFU} /$ cell (closed triangles), at a $\mathrm{MOI}$ of 1 (closed circles) and at a MOI of 10 (closed squares) for $1 \mathrm{~h}$, before washing vigorously. Virus titers obtained $48 \mathrm{~h}$ p.i. from Vero cells exposed to HSV-2 at a MOI of 1 and processed as for U937 cells, are also reported (open triangles). (A) Total virus yield, evaluated using cell extracts obtained by freezing and thawing the cells twice in their culture medium. (B) Extracellular virus, evaluated by using cell free supernatants from infected cultures. Similar results were obtained in five independent experiments. (C) Comparison of amounts of protein synthesis in U937 and in Vero cells following infection with HSV-2, $24 \mathrm{~h}$ p.i.. For protein synthesis measurements, cells were kept for 15 min in methionine-deprived culture medium, before labeling with $20 \mu \mathrm{Ci} / \mathrm{ml}{ }^{35}$ S ]methionine for $45 \mathrm{~min}$. After labeling, cells were wahsed three times with PBS and lysed. Aliquots of lysates were then precipitated with TCA and the radioactivity incorporated was determined using a $\beta$-counter. Results are expressed as mean percentages in respect to uninfected controls, obtained from two independent experiments. (D) Kinetic of protein synthesis in U937 following infection with HSV-2. Protein synthesis measurement was performed as in (C). Results are expressed as cumulative mean percentages of uninfected control \pm S.D., calculated from two independent experiments performed in duplicate

\section{Cycloheximide and moderate exposure to UV do not abrogate HSV-2-induced apoptosis in monocytoid cells}

Finally, we were interested in obtaining more information on the mechanisms involved in cell death by apoptosis caused by HSV-2. To this purpose we tested whether the addition of cycloheximide $(\mathrm{CHX})$ could interfere with the induction of apoptosis by HSV-2. CHX by itself, at $50 \mu \mathrm{g} / \mathrm{ml}$, induced a well detectable level of apoptosis in untreated U937 cells, as shown by the DNA-electrophoresis-on-agarose-gel technique (Figure 6a, lane 2). In fact, the U937 cell line has previously been shown to be sensitive to $\mathrm{CHX}$-induced apoptosis (Martin et al, 1990). Detection of apoptosis was not substantially modified in U937 cells treated with the same dose of $\mathrm{CHX}$, following HSV-2 infection (Figure 6a, lane 3). Conversely, uninfected Vero cells were resistant to $\mathrm{CHX}$-induced apoptosis (Figure 6a, lane 4); furthermore, HSV-2 infection did not cause apoptosis in Vero cells treated with $\mathrm{CHX}$ at $50 \mu \mathrm{g} / \mathrm{ml}$ (Figure 6a, lane 5) or at lower doses (data not shown). However, the addition of $\mathrm{CHX}$ at $1 \mu \mathrm{g} / \mathrm{ml}$, a dose which by itself induced quite a low level of apoptosis in U937 cells while still completely suppressing protein sysnthesis, did not prevent HSV-2-induced apoptosis as evaluated by fluorescence microscopy following acridine-orange-staining (Figure $6 \mathrm{~b}$ ). Indeed, pre-treatment with $\mathrm{CHX} 1 \mathrm{~h}$ prior to infection seemed to enhance virusinduced apoptosis, detected at levels more than double those of untreated infected cultures, suggesting that de novo host or viral protein synthesis is dispensable in triggering apoptosis and that one or more virion-associated molecules could be responsible for this phenomenon. This hypothesis is consistent with the fact that HSV-2-induced apoptosis in U937 cells was highly dependent on the dose of virus inoculum. We have further investigated this possibility by using UV irradiation of the virus and by comparing the inactivation of infectivity with that of apoptosis-inducing activity. As shown in Figure 7, both 
infectivity and apoptosis-inducing activity were strongly suppressed by prolonged exposure to UV light. However, when the virus was exposed to UV for a shorter time, we observed no strict link between the suppression of apoptosis-inducing activity and that of infectivity. In fact, only slight reductions of apoptosis were observed following infection with virus inocula in which infectivity was reduced a hundredfold by UV treatment.

\section{a}

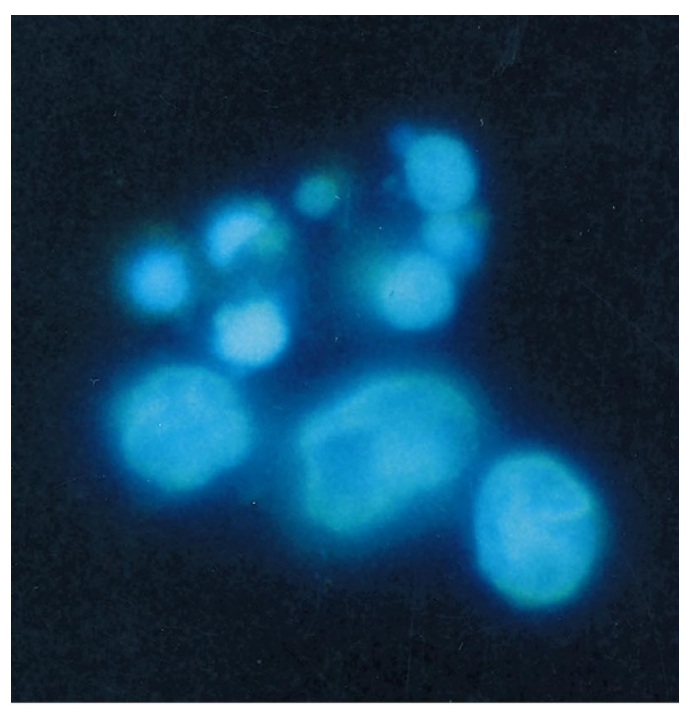

b

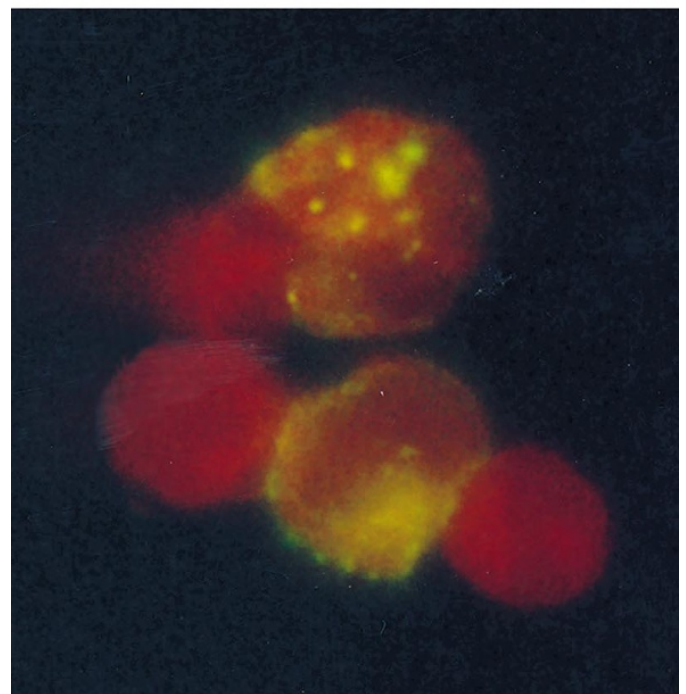

Figure 5 Double-fluorescence microscopy analysis of HSV-2-infected U937 cells. Cells infected with HSV-2 at a MOI of $10 \mathrm{PFU} /$ cell were analyzed $24 \mathrm{~h}$ p.i., after dual staining for simultaneous detection of apoptotic cells and virusprotein-positive cells. (A) Staining with the fluorescent DNA-binding dye Hoechst, showing two cells with typical characteristics of advanced apoptosis, with nuclei present as a group of featureless, bright, spherical beads. (B) Staining with a monoclonal antibody specific for HSV-2 glycoprotein D, followed by FITC-conjugated goat anti-mouse IgG. One of the two apoptotic cells colocalizes virus protein. Another HSV-2-protein-positive cell does not show apoptosis characteristics. Magnification $(\times 400)$

\section{Indomethacin treatment reduces induction of apoptosis by HSV-2}

The cyclic pathway from arachidonate to eicosanoids could be one of the biochemical mechanisms involved in apoptosis caused by virus infection of cells of the monocyte/macrophage lineage, as we have shown with HIV-infected macrophages, in an experimental model in vitro (Mastino et al, 1993). Thus, we added indomethacin $1 \mathrm{~h}$ before infection of U937 with HSV-2. When indomethacin $\left(10^{-6} \mathrm{M}\right)$ was added to HSV-2-infected cultures, the percentage of apoptotic cells was significantly reduced by about $40 \%$ compared with untreated infected cultures $24 \mathrm{~h}$ p.i. (Figure $8 \mathrm{a}$ ). The same dose of indomethacin did not significantly modify apoptosis in control uninfected U937 cells.

\section{HSV-2-induced apoptosis in monocytoid cells is associated with a down-modulation of Bcl-2}

Expression of the $b c l-2$ gene is one of the mechanisms associated with modulation of cell apoptosis during viral infections (Akbar et al, 1993; Boudet et al, 1996; Levine et al, 1996; Olsen et al, 1996). Using flow cytometry, we thus analyzed Bcl-2 protein cellular level in U937 following HSV-2 infection. Results showed a clear virus-dose-dependent down-modulation of $\mathrm{Bcl}-2$ in a high proportion of the cells $48 \mathrm{~h}$ following the infection at a MOI of $10 \mathrm{PFU} / \mathrm{cell}$, i.e. in experimental conditions able to induce high level of apoptosis (Figure 8b). Bcl-2 down-regulation could, therefore, be considered to be reasonably involved in HSV-induced apoptosis of monocytoid cells.

\section{Discussion}

Herpes simplex viruses are known to cause a lytic cycle of infection or persistently latent infection in cells of specific histological types. The mechanism of virus persistence is not fully understood but inhibition of apoptosis has been hypothesized to be one of the phenomena involved (Chou and Roizman, 1992). For this reason, authors' interest has been mainly centered on the suppression of apoptosis by HSVs and on the viral molecules responsible for this inhibitory phenomenon (Leopardi and Roizman, 1996; Koyama and Miwa, 1997). However, isolated reports indicated the apoptosis-inducing capacity of HSVs in vitro as well as in vivo (Tropea et al, 1995; Geiger et al, 1995). Our results confirm the ability of viruses belonging to Herpesviridae family to induce apoptosis in infected cells and allow us to extend this observation to HSV-2 infection in vitro. We demonstrated a virus inoculum-dependent and time-dependent induction of apoptosis in U937, showing that apoptosis in this cell line, as a result of exposure to the virus, was not restricted to HSV-2 but was common to the herpes simplex viruses. However, we must note that levels of \% apoptosis obtained using the HSV1 ' $F$ ' strain were lower than those obtained using HSV-2. Nevertheless, utilizing an HSV-1 strain of different origin, the laboratory strain EB, we obtained percentages of apoptosis closer to those obtained with the HSV-2 strain ' $G$ ', but consistently lower (data not shown). Thus, differences 
between the HSV-2 strain 'G' and the HSV-1 strain 'F', should be attributed part to 'type-specific' and part to 'strain-specific' characteristics.

a

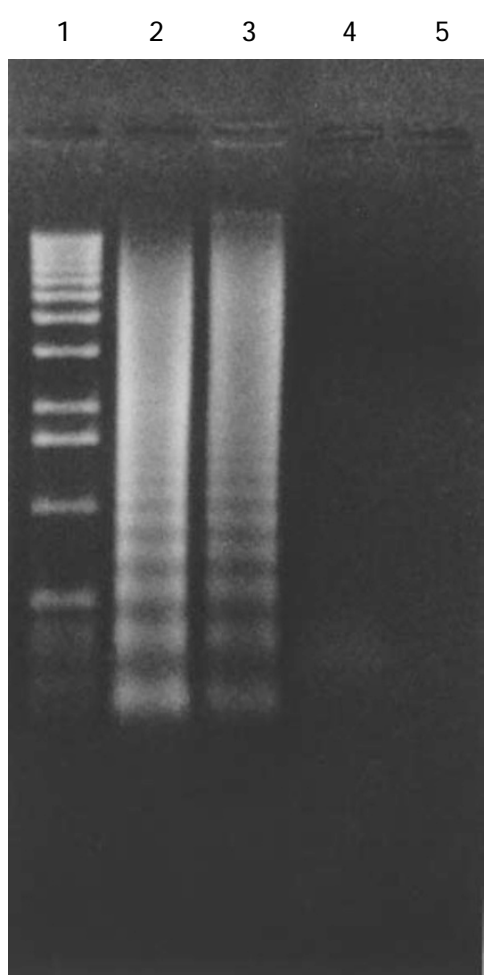

b

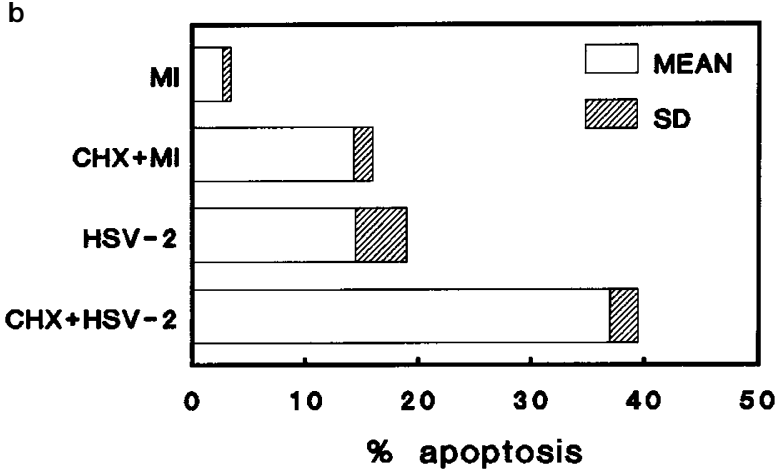

Figure 6 Effect of cycloheximide on induction of apoptosis by HSV-2 infection. (A) Cells were pretreated with cycloheximide (CHX, $50 \mu \mathrm{g} / \mathrm{ml})$ for $1 \mathrm{~h}$ and then mock-infected (U937, lane 2; Vero, lane 4) or infected with HSV-2 at a $\mathrm{MOI}$ of $10 \mathrm{PFU} / \mathrm{cell}$ (U937, lane 3; Vero lane 5). Cells were vigorously washed three times $1 \mathrm{~h} \mathrm{p.i.} \mathrm{and} \mathrm{resuspended} \mathrm{in} \mathrm{fresh} \mathrm{growth} \mathrm{medium} \mathrm{again}$ with or without $50 \mu \mathrm{g} / \mathrm{ml}$ of cycloheximide. After $24 \mathrm{~h}$ at $37^{\circ} \mathrm{C}$ cells were collected, DNA extracted, electrophoresed on $0.8 \%$ agarose gel and visualized by staining with ethidium bromide and by UV light transillumination. Lane 1: m.w. marker of a $1 \mathrm{~Kb}$ ladder DNA. (B) U937 cells were pretreated with cycloheximide $(\mathrm{CHX}, 1 \mu \mathrm{g} / \mathrm{ml})$ for $1 \mathrm{~h}$ or were left untreated and then mockinfected (MI) or infected with HSV-2 at a MOI of $10 \mathrm{PFU} / \mathrm{cell}$ (HSV-2). Cells were vigorously washed three times $1 \mathrm{~h}$ p.i. and resuspended in fresh growth medium with or without $1 \mu \mathrm{g} / \mathrm{ml}$ of cycloheximide. At $24 \mathrm{~h} \mathrm{p.i.,} \mathrm{percentage} \mathrm{of}$ apoptosis was evaluated by fluorescence microscopy analysis following staining with acridine orange. Over 600 cells, including apoptotic ones, were counted for each sample. Results are expressed as cumulative mean values + S.D. obtained from four independent experiments. Statistical analysis by paired Student's $t$ test. CHX+HSV-2 vs all other groups, $P<0.001$; HSV-2 vs $\mathrm{MI} P=0.011 ; \mathrm{CHX}+\mathrm{Ml}$ vs $\mathrm{MI}, P<0.001 ; \mathrm{HSV}-2$ vs $\mathrm{CHX}+\mathrm{Ml}=\mathrm{NS}$
Results of double fluorescence staining indicate that apparently intact cells as well as apoptotic cells were infected. Similarly, apoptosis was observed both in virusprotein-positive and in virus-protein-negative cells. Different hypotheses could account for this observation. One is that in some cells morphological changes leading to apoptosis could occur very rapidly after infection, not allowing the further progression of virus replication and the consequent production of detectable amounts of virus specific glycoproteins. On the other hand, it may be possible that part of the cells could be induced to die by apoptosis indirectly, without being infected by the virus. In this case cell death could be caused by exposure to apoptosisinducing cellular or viral products released by infected cells. Interestingly, a similar indirect effect was demonstrated by us using a different experimental model of virus infection in monocytic/macrophage cells in vitro (Mastino et al, 1993).

\section{A INACTIVATION OF APOPTOTIC ACTIVITY}

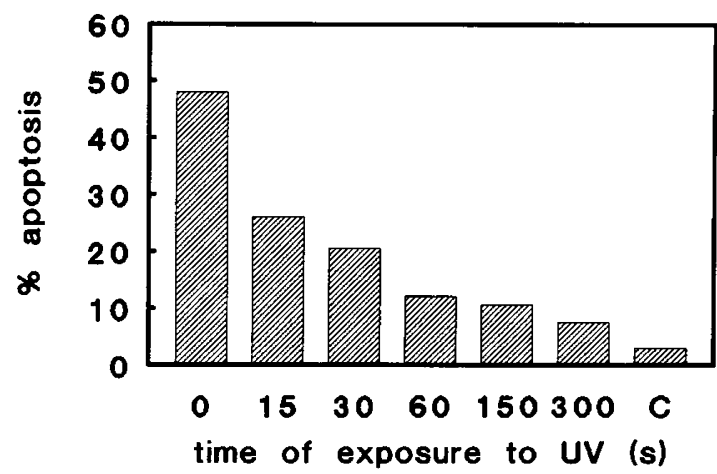

B

INACTIVATION OF INFECTIVITY

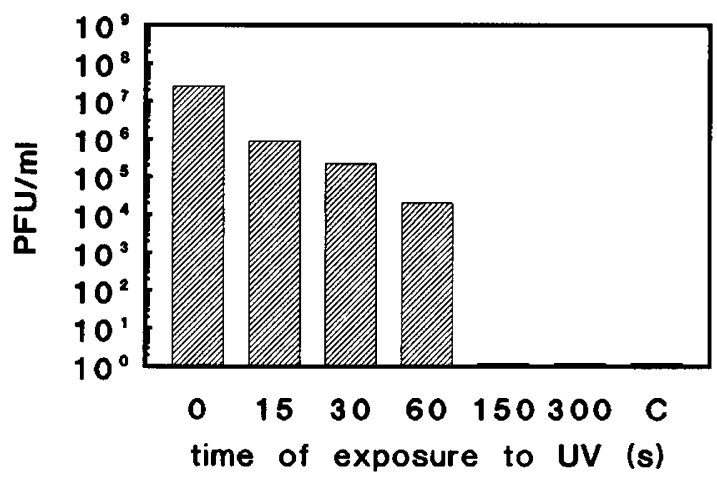

Figure 7 Effect of UV treatment on inactivation of infectivity and of apoptotic activity by HSV-2. (A) Percentage apoptosis induced in U937 cells, evaluated as described in Figure 5, following different times of exposure of HSV-2 to UV. (B) Virus titrations by plaque assay on Vero cells, following different times of exposure of HSV-2 to UV. Note that infectivity, expressed as PFU/ml, has been represented on a logarithmic scale while percentage of apoptosis has been represented on a linear scale. Data from a representative experiment are shown; similar results were obtained in two other experiments 
Our results indicate some of the mechanisms correlated with HSV-2-induced apoptosis, opening the way to identifying the viral components involved. We did not observe a strict quantitative link between the suppression of apoptosis-inducing activity and that of infectivity as shown by experiments with UV treatment. This fact

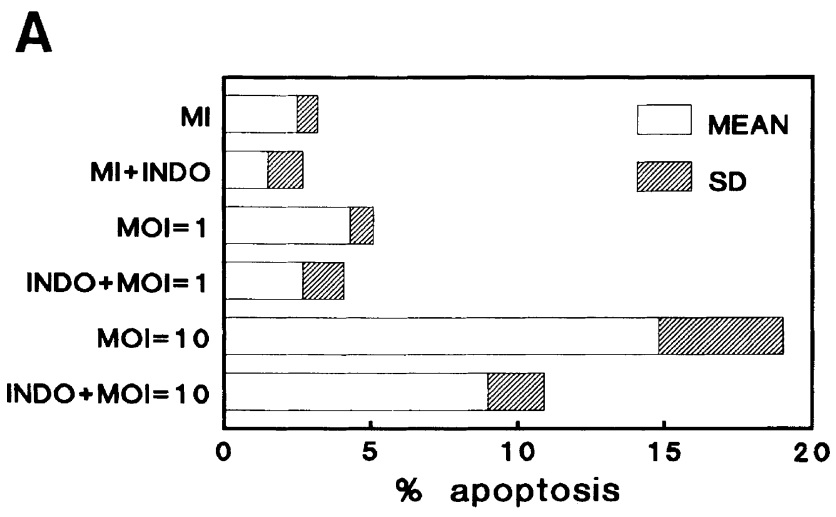

B
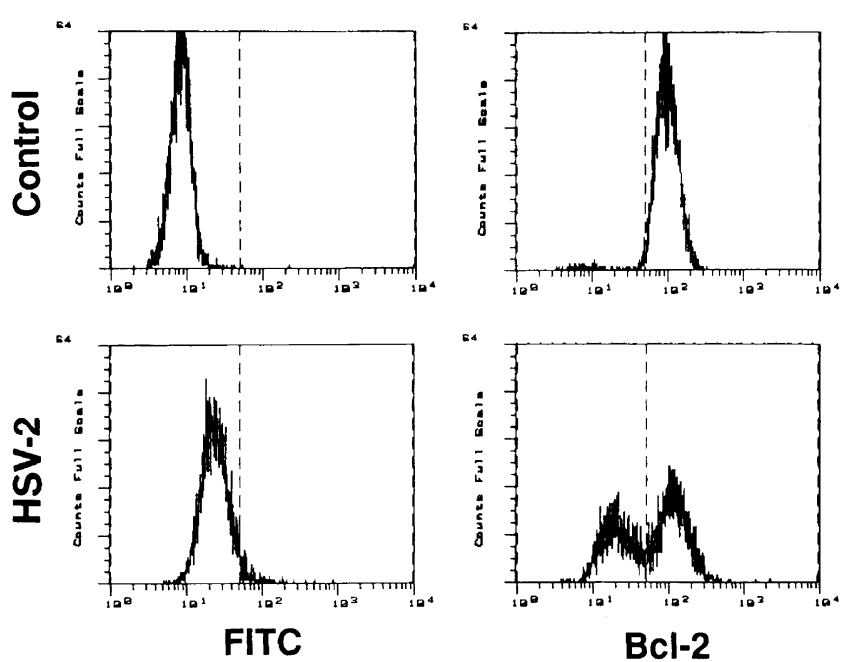

Figure 8 Biochemical and molecular events involved in apoptosis induced by HSV-2 on U937 cells. (A) U937 cells were treated with $10^{-6} \mathrm{M}$ indomethacin (INDO) for $1 \mathrm{~h}$ or were left untreated and then mock-infected (MI) or infected with HSV-2 at a $\mathrm{MOI}$ of $1 \mathrm{PFU} /$ cell or at a $\mathrm{MOI}$ of $10 \mathrm{PFU} /$ cell. Cells were vigorously washed three times $1 \mathrm{~h}$ p.i. and resuspended in fresh growth medium with or without INDO. At $24 \mathrm{~h}$ p.i., percentage of apoptosis was evaluated as described in Figure 5. Results, obtained in three independent experiments, are expressed as mean values+S.D. Statistical analysis by paired Student's $t$ test. MOI=10 vs all other groups, $P<0.05 ; \mathrm{MOI}=1 \mathrm{vs}$ all other groups, $P<0.05$. (B) For flow cytometry analysis of $\mathrm{Bcl}-2$ protein, cells from mock-infected (Control) or HSV-2 (10 PFU/ml) infected cultures (HSV-2), $48 \mathrm{~h}$ p.i. were fixed in $4 \%$ paraformaldehyde in PBS for $20 \mathrm{~min}$ at room temperature and permeabilized in $0.1 \%$ Triton X-100 in PBS with $0.1 \%$ FCS for 5 min. After washing twice in PBS with $3 \%$ FCS, permeabilized cells were incubated with a rabbit polyclonal anti-human bcl-2 (Bcl-2) or irrelevant control antibodies (FITC) for $30 \mathrm{~min}$ on ice and washed twice in PBS. Anti-bcl-2-stained cells were incubated with a FITC-conjugated goat anti-rabbit lg antibody at a $1: 30$ dilution for $30 \mathrm{~min}$ on ice and then rinsed twice in PBS with $0.1 \%$ sodium azide. The dashed lines indicate the boundaries between the peaks of negative and positive cells which were arbitrarily set for control samples and maintained in all the corresponding anti-bcl-2/FITC-anti-rabbit stained samples. Similar results were obtained in three independent experiments indicates that virus particles in which full infectivity was abrogated, were still able to induce apoptosis, confirming the hypothesis that virion-associated molecules are involved in apoptosis induced by HSV-2. Interestingly, a similar uncoupling of suppression of infectivity and suppression of protein synthesis following HSV-2 irradiation with UV was observed many years ago (Fenwick and Walker, 1978), demonstrating that structural proteins of herpesviruses, can shut off cellular protein synthesis. Therefore, virus-induced apoptosis in U937 cells could be linked, at least in part, to a herpesvirus-induced shut-off of protein synthesis in host cells. Moreover, UV-inactivated bovine herpesvirus 1 has recently been demonstrated to maintain its ability to induce apoptosis in bovine peripheral blood mononuclear cells (Hanon et al, 1996). In addition, the fact that the cyclooxigenase inhibitor indomethacin reduced apoptosis caused by HSV-2 indicates that eicosanoid-release by U937 cells could be one of the mechanisms involved in virus-induced apoptosis. Prostaglandin E2 (PGE2) and other agents able to activate protein kinase $A$, have been shown to induce apoptosis in vitro (McConkey et al, 1990) as well as in vivo (Mastino et al, 1992). However, we were not able to reproduce apoptosis in U937 cells by direct exposure of the cultures to PGE2 (data not shown), suggesting that mechanisms other than PGE2 production could be involved in indomethacin protection against HSV-2-induced apoptosis. Moreover, indomethacin treatment did not affect virus yield in monocytoid cells (data not shown). This excludes the possibility that indomethacin inhibitory action could be due to a protective effect against virus infection and/or replication. Interestingly, indomethacin has recently been shown to protect against stress-induced cellular damage through the modulation of heat shock factor 1 (Lee et al, 1995). Considering that HSV infection could result in elevated expression of heat shock proteins (La Thangue et al, 1984), protection by indomethacin against virusinduced apoptosis could be referred to a similar action.

In conclusion, our data demonstrate that the same HSV strains able to produce a lytic cycle of infection, without exhibiting any evidence of apoptosis in a fully permissive cell line, induced features characteristic of apoptosis in at least monocytoid cells. This result suggests that host cell factors could play a critical role in regulating the balance between the pro-apoptotic and the anti-apoptotic virus poential. Thus, even in the absence of any modification of the HSV genome, cells of specific histological types infected with HSV-2 or HSV-1 undergo a peculiar type of infection which differs from the classical productive-lytic and persistent-latent ones. In terms of kinetic and absolute quantity of virus production this kind of infection is located halfway between productive-lytic and persistent-latent infections and we propose identifying it as 'apoptotic infection', according to the specificity of the cytophatic effect observed. Modulation of apoptosis could be one of the mechanisms by which the final fate of target cells could be regulated. In the case of inhibition of apoptosis, survival as persistently infected cells (nervous tissue) or death by necrosis during initial or recurrent infection (mucosal tissue) could occur; alternatively, in the case of induction, cell 
death by apoptosis in the absence of an inflammatory reaction and in the presence of a limited and delayed spread of the virus could predominate (myeloid and lymphoid tissues). In fact, studies in progress in our laboratory indicate that induction of apoptosis by HSV is not restricted to monocytoid cells, but could also be observed in various cell lines of lymphoid lineage (unpublished data). HSV has been found to adopt specific active strategies in order to evade immune cell recognition (York et al, 1994). Apoptosis of lymphomonocytes could be an additional mechanism by which the virus could control host immune response. Our results could, therefore, contribute to the clarification of the mechanisms by which herpesviruses exert their pathological action. Further investigations are necessary in order to better understand the role of apoptotic cell death during HSV-2 infection and the mechanisms involved in this phenomenon.

\section{Materials and Methods}

\section{Viruses, cells and treatments}

A ' $G$ ' strain of HSV-2, originally obtained from the American Type Culture Collection, was used in most of the experiments. Virus stocks were produced by infecting at low multiplicity cultures of Vero (African green monkey kidney) cells propagated in MEM (HyClone Europe, Cramlington, UK) containing $10 \%$ fetal calf serum (FCS) (Life Technologies, Gaithersburg, $\mathrm{MD}$ ) at $37^{\circ} \mathrm{C}$ in a $\mathrm{CO}_{2}$ incubator. Virus stocks gave a titer of approximately $1 \times 10^{7} \mathrm{PFU} / \mathrm{ml}$ in Vero cells and were collected and stored in aliquots at $-80^{\circ} \mathrm{C}$. For experimental infection approximately $5 \times 10^{5} \mathrm{U} 937$ cells or confluent monolayers of Vero cells in 24/well plates, were inoculated, $24 \mathrm{~h}$ after cultures had been split, in complete medium (CM) with HSV-2 at different PFU/cell ratios and incubated at $37^{\circ} \mathrm{C}$ in $5 \% \mathrm{CO}_{2}$. CM consisted of RPMI 1640 (HyClone Europe) for U937 cells or MEM for Vero cells respectively, supplemented with $2 \mathrm{mM} \mathrm{L-glutamine,} 100$ units/ml penicillin, $100 \mu \mathrm{g} /$ $\mathrm{ml}$ streptomycin and $10 \%$ heat-inactivated FCS. In experiments requiring inactivated virus, virus suspensions were placed in petri dishes $(35 \mathrm{~mm})$ and, in constant agitation, a volume of $1 \mathrm{ml}$ was exposed, for varing times, to UV light at an intensity of $30 \mathrm{~W}$ from a germicidal lamp situated $10 \mathrm{~cm}$ above the sample. For mock-infection, target cells were exposed to control cellular extracts collected from uninfected Vero cells, manipulated and then stored in the same way as those containing the virus. Infected cultures were vigorously washed three times $1 \mathrm{~h}$ post-infection and the inocolum was replaced by fresh growth medium. Infected and control cells were grown at $37^{\circ} \mathrm{C}$ in $\mathrm{CM}$. In some experiments HL-60, as target cells, and in others HSV-1, as virus inoculum (Strain ' $F$ ', originally obtained from ATCC, or strain EB, kindly provided by C.F. Perno, University of Rome 'Tor Vergata'), produced and utilized as described for HSV-2, were also used. Indomethacin $\left(10^{-6} \mathrm{M}\right.$; Sigma, St. Louis, MO) was added to the cultures $1 \mathrm{~h}$ before infection. Cycloheximide (Sigma) was utilized at $1-50 \mu \mathrm{g} / \mathrm{ml}$, i.e. at concentrations which completely inhibited both host and virus protein synthesis in U937 and Vero cells.

\section{Evaluation of apoptosis}

At different times following infection, the percentage of apoptotic cells from infected or control cultures was evaluated using different techniques. Morphological analysis was performed following staining with acridine orange or Hoechst chromatin dyes (Sigma). Over 600 cells including those showing typical apoptotic characteristics were counted by using a Biomed fluorescence microscope (Leitz, Wetzlar, Germany). The percentage of apoptotic cells was calculated as follows:

$$
\% \text { apoptosis }=\frac{\text { Total no. of cells with apoptotic nuclei }}{\text { Total no. of cells counted }} \times 100
$$

Flow cytometry analysis of isolated nuclei was performed using a method described for murine thymocytes (Nicoletti et al, 1991), with minor modifications. Briefly, cells were collected from culture wells and washed twice in $\mathrm{CM}$ in $12 \times 75 \mathrm{~mm}$ polypropylene tubes (Falcon, Becton Dickinson Labware, Lincoln Park, NJ). Cell pellet was gently resuspended at room temperature in $1 \mathrm{ml}$ of hypotonic solution consisting of: propidium iodide $50 \mu \mathrm{g} / \mathrm{ml}$ (Becton Dickinson, Mountain View, CA), $0.1 \%$ sodium citrate (Merck, Darmstadt, Germany) and $1 \%$ Triton X-100 (Sigma) in distilled water. The tubes were placed at $4^{\circ} \mathrm{C}$ in the dark overnight. Flow cytometry analysis was subsequently performed using a FACscan flow cytometer (Beckton Dickinson). A correct threshold value was experimentally selected in order to exclude the majority of cell debris. Data collection was gated using adequate values of forward- and side-angle scatter to exclude remaining cell debris and large nuclei aggregates, and to include nuclei from apoptotic, necrotic and living cells. For each sample, 5000 events were acquired. Analysis of DNA fragmentation was carried out at single cell level using the TUNEL technique or at culture level by gel electrophoresis. Labeling of DNA strand breaks with fluorescein-dUTP by terminal deoxynucleotidyl transferase was performed using a commercial kit ('In situ cell death detection kit fluorescein'; Boehringer Mannheim, Mannheim, Germany) according to the manufacturer's instructions. Positive control consisted of cells treated with DNase 1 $(1 \mathrm{mg} / \mathrm{ml})$ for $10 \mathrm{~min}$ at room temperature. Analysis of labeled cells was performed both by fluorescence microscopy or flow cytometry. For flow cytometry, labeled cells were acquired and analyzed on a FACscan (Becton-Dickinson) using the FACscan program (BectonDickinson), following adequate gating by scatter parameters in order to exclude cell debris and large cell aggregates, and to include presumably apoptotic, necrotic and living cells. For each sample, 5000 events were acquired. For analysis of DNA fragmentation by gel electrophoresis, cells were washed in PBS and then lysed in $0.5 \mathrm{ml}$ of lysis buffer (10 mM Tris, pH 7.4, 1 mM EDTA, pH 8.0, 0.2\% Triton X$100)$ containing proteinase $\mathrm{K}(100 \mu \mathrm{g} / \mathrm{ml})$ for $1 \mathrm{~h}$ at room temperature. Samples were then centrifuged at 13000 r.p.m. for $15 \mathrm{~min}$. DNA in the supernatants was precipitated with $5 \mathrm{~N} \mathrm{NaCl}$ in 1 volume of isopropanol for $1 \mathrm{~h}$ at $-20^{\circ} \mathrm{C}$. The DNA precipitates were recovered by centrifugation at 13000 r.p.m., air dried for $15 \mathrm{~min}$ at room temperature, resuspended in TE buffer $(10 \mathrm{mM}$ Tris, $\mathrm{pH} 7.4,1 \mathrm{mM}$ EDTA, pH 8.0) containing RNase $(100 \mu \mathrm{g} / \mathrm{ml})$ and incubated for $1 \mathrm{~h}$ at $37^{\circ} \mathrm{C}$. Before electrophoresis, loading buffer ( $2 \%$ SDS, $15 \mathrm{mM}$ EDTA, $0.25 \% \mathrm{w} / \mathrm{v}$ bromophenol blue, $50 \% \mathrm{v} / \mathrm{v}$ glycerol) was added to each sample in at a ratio of $1: 5$.

\section{Virus titration}

Extracellular virus or total virus (cell-associated plus extracellular virus) were titrated in supernatants from cultures or in aliquots of cell extracts obtained from cultures by freezing and thawing, respectively. Virus titers were determined by standard plaque assay on Vero cells. HSV-2 in U937 cells was also detected by using fluorescence microscopy and a commercial HSV-2-specific monoclonal antibody (Kallestad, Sanofi Diagnostics Pasteur, Chaska, MN) or a monoclonal antibody which recognizes HSV-2 glycoprotein D (LP2), kindly provided by Dr. S. Bacchetti (Hamilton, Canada). 


\section{Protein synthesis and PAGE}

The kinetics and the amounts of protein synthesis in uninfected or HSV-2-infected U937 and Vero cells were studied. At different times after infection cells were labeled with $20 \mu \mathrm{Ci} / \mathrm{ml}^{35} \mathrm{~S}$ methionine (Amersham, Amersham Place, Buckinghamshire, UK) for $45 \mathrm{~min}$. For protein synthesis measurements, cells were kept for $15 \mathrm{~min}$ in methionine-deprived culture medium, prior to labeling. After labeling, cells were washed three times with PBS and lysed in lysis buffer ( $2 \%$ SDS, $10 \%$ glycerol, $0.001 \%$ bromophenol blue, $0.1 \mathrm{M}$ dithiothreitol, $0.625 \mathrm{M}$ Tris- $\mathrm{HCl} \mathrm{pH} \mathrm{6.8).} \mathrm{Aliquots} \mathrm{of} \mathrm{lysates} \mathrm{were} \mathrm{then} \mathrm{precipitated}$ with TCA and the radioactivity incorporated was determined using a $\beta$ counter (LKB Wallac, Turku, Finland) as previously described (Santoro et al, 1982). Comparable amounts of samples, according to radioactivity counts, were analyzed by SDS-PAGE in a vertical gel apparatus as described previously (Santoro et al, 1989).

\section{Flow cytometry analysis of $\mathrm{Bcl}-2$ protein}

Cells were fixed in $4 \%$ paraformaldehyde in PBS $(\mathrm{pH} 7.4)$ for $20 \mathrm{~min}$ at room temperature and permeabilized in $0.1 \%$ Triton $\mathrm{X}$ 100 in PBS with $0.1 \%$ FCS for 5 min. After washing twice in PBS with 3\% FCS, permeabilized cells were incubated with a rabbit polyclonal anti-human bcl-2 (Pharmingen, San Diego, CA) or with irrelevant control antibodies for $30 \mathrm{~min}$ on ice and washed twice in PBS. Anti-bcl-2-stained cells were incubated with a FITCconjugated goat anti-rabbit Ig antibody (Harlan Sera-Lab, Crawley Down, UK) at a 1:30 dilution for $30 \mathrm{~min}$ on ice and then rinsed twice in PBS with $0.1 \%$ sodium azide. Stained cells were acquired and analyzed on a FACScan (Becton-Dickinson) as previously described for the TUNEL technique.

\section{Statistical analysis}

Analysis of data was performed using the SPSS statistical software system (version 6.0 for Windows, SPSS, Chicago, IL). Comparisons of means were carried out using the Duncan test, as a multiple range test, or the Student's $t$ test for paired samples.

\section{Acknowledgements}

We thank Prof. Enrico Garaci for his support, insights and helpful discussions throughout this work; Dr. Silvia Bacchetti for kindly providing LP2 monoclonal antibody; Alison Inglis, B.A., for her helpful linguistic appraisal. This work was supported by M.U.R.S.T. 40\% (A. Pernice) and $60 \%$ (A. Mastino) and by C.N.R. Special Projects 'Aging' and 'Cell cycle and apoptosis' (A. Mastino).

\section{References}

Akbar AN, Borthwick N, Salmon M, Gombert W, Bofill M, Shamsadeen N, Pilling D, Pett S, Grundy JE and Janossy G (1993) The significance of low bcl-2 expression by CD45RO T cells in normal individuals and patients with acute viral infections. The role of apoptosis in T cell memory. J. Exp. Med. 178: 427-438

Arends MJ and Wyllie AH (1991) Apoptosis: mechanism and role in pathology. Int. Rev. Exp. Pathol. 32: 223-254

Boudet F, Lecoeur Hand Gougeon M (1996) Apoptosis associated with ex vivo downregulation of $\mathrm{Bcl}-2$ and up-regulation of Fas in potential cytotoxic CD8+ T lymphocytes during HIV infection. J. Immunol. 156: 2282-2293

Chou J and Roizman B (1992) The $\gamma 134.5$ gene of herpes simplex virus 1 precludes neuroblastoma cells from triggering total shutoff of protein synthesis characteristic of programmed cell death in neuronal cells. Proc. Natl. Acad. Sci. USA. 89: $3266-3270$
Feng C, Kulka M and Aurelian L (1993) NF- $\kappa$ B-binding proteins induced by HSV-1 infection of $U 937$ cells are not involved in activation of human immunodeficiency virus. Virology. 192: $491-500$

Fenwick ML and Walker MJ (1978) Suppression of the synthesis of cellular macromolecules by herpes simplex virus. J. Gen. Virol. 41: $37-51$

Geiger KD, Gurushanthaiah D, Howes EL, Lewandowski GA, Reed JC, Bloom FE and Sarvetnick NE (1995) Cytokine-mediated survival from lethal herpes simplex virus infection: role of programmed neuronal death. Proc. Natl. Acad. Sci. USA. 92: 3411-3415

Ghibelli L, Maresca V, Coppola S and Gualandi G (1995) Protease inhibitors block apoptosis at intermediate stages: A compared analysis of DNA fragmentation and apoptotic nuclear morphology. FEBS Lett. 377: 9-14

Hanon E, Vanderplasschen A, Lyaku J, Keil G, Denis M and Pastoret P (1996) Inactivated bovine herpesvirus 1 induces apoptotic death of mitogen stimulated bovine peripheral blood mononuclear cells. J. Virol. 70: 4116-4120

Kerr JFR, Wyllie AH and Currie AR (1972) Apoptosis: A basic biological phenomenon with wide-ranging implication in tissue kinetics. Brit. J. Cancer. 26: $239-257$

Koyama AH and Miwa Y (1997) Suppression of apoptotic DNA fragmentation in herpes simplex virus type 1-infected cells. J. Virology. 71: 2567-2571

La Thangue NB, Shriver K, Dawson C and Chan WL (1984) Herpes simplex virus infection causes the accumulation of a heat shock protein. EMBO J. 3:267-277

Lee BS, Chen J, Angelidis C, Jurivich D and Morimoto RI (1995) Pharmacological modulation of heat shock factor 1 by antiinflammatory drugs results in protection against stress-induced cellular damage. Proc. Natl. Acad. Sci. USA. 92: 72077211

Leopardi R and Roizman B (1996) The herpes simplex virus major regulatory protein ICP4 blocks apoptosis induced by the virus or by hyperthermia. Proc. Natl. Acad. Sci. USA. 93: 9583-9587

Levine B, Goldman JE, Jiang HH, Griffin DE and Hardwick JM (1996) Bcl-2 protects mice against fatal alphavirus encephalitis. Proc. Natl. Acad. Sci. USA. 93:48104815

Marin SJ, Lennon SV, Bonham AM and Cotter TG (1990) Induction of apoptosis (Programmed cell death) in human leukemic HL-60 cells by inhibition of RNA or protein synthesis. J. Immunol. 145: 1859-1867

Mastino A, Piacentini M, Grelli S, Favalli C, Autori F, Tentori L, Oliverio Sand Garaci E (1992) Induction of apoptosis in thymocytes by prostaglandin E2 in vivo. Dev. Immunol. 2: 263-271

Mastino A, Grelli S, Piacentini M, Oliverio S, Favalli C, Perno CF and Garaci E (1993) Correlation between induction of lymphocyte apoptosis and prostaglandin E2 production by macrophages infected with HIV. Cell. Immunol. 152: 120-130

McCabe MJ jr and Orrenius S (1992) Deletion and depletion: the involvement of viruses and environmental factors in T-lymphocyte apoptosis. Lab. Investigation 66: $403-406$

McConkey DJ, Orrenius S and Mikael J (1990) Agents that elevate cAMP stimulate DNA fragmentation in thymocytes. J. Immunol. 145: 1227-1230

Nicoletti I, Migliorati G, Pagliacci MC, Grignani F and Riccardi C (1991) A rapid and simple method for measuring thymocyte apoptosis by propidium iodide staining and flow cytometry. J. Immunol. Methods 139: 271-279

Olsen CW, Kehren JC, Dybdahl-Sissoko NRand Hinshaw VS(1996) bcl-2 Alters influenza virus yield, spread, and hemagglutinin glycosylation. J. Virol. 70:663-666

RazviES andWelshRM (1995) Apoptosis in viral infections. Adv. Virus Res. 45:1-60

Roizman B and Sears AE (1996) Herpes simplex viruses and their replication. In Field's Virology, third edition, Fields BN, Knipe DM, Howley P, Chanock RM, Hirsh MS, Melnick JL, Monath TP and Roizman B, eds. (Philadelphia: LippincottRaven Publishers) pp. 2231-2295

Santoro MG, Jaffe BM, Garaci E and Esteban M (1982) Antiviral effect of prostaglandins of the A series: inhibition of vaccinia virus replication in cultured cells. J. Gen. Virol. 63: 435-440

Santoro MG, Amici C, Elia G, Benedetto A and Garaci E (1989) Inhibition of virus protein glycosylation as the mechanism of the antiviral action of prostaglandin $A$ in Sendai virus-infected cells. J. Gen. Virol. 70: 789-800

Shen Y and Shenk TE (1995) Virus and apoptosis. Curr. Opin. Gen. Dev. 5: 105-111

Tropea F, Triano L, Monti D, Lovato E, Malorni W, Rainaldi G, Mattana P, Viscomi G, Ingletti MC, Portolani M, Cermelli C, Cossarizza A and Franceschi C (1995) Sendai virus and herpes virus type 1 induce apoptosis in human peripheral blood mononuclear cells. Exp. Cell. Res. 218: 63-70

York IA, Roop C, Andrews DW, Riddell SR, Graham FL and Johnson DC (1994) A cytosolic herpes simplex virus protein inhibits antigen presentation to CD8+ $T$ lymphocytes. Cell 77: 525-535 